health study of patients on prescription opioid pain medications in Erie county medical center and local out patient clinic. The electronic data from the hospital records and Outpatient were collected, merged and de identified. The database was saved in a protected environment and made accessible to researchers through a secure login. The data was queried for the number of patients with diabetes. The glycohemoglobin levels were collected and then the analysis was made RESULTS/ANTICIPATED RESULTS: It was found that only 63 of the 89 patients with DPN and 156 of the 570 patients without DPN had any measurement of HbAlc in our data. It was found that 86 out of 156 patients without DPN had suboptimal glycemic control with a glycohemoglobin level $>7 \%$ while 36 out of 63 patients with DPN had a glycohemoglobin $>6.7 \%$. The odds of patients with DPN having poor glycemic control is 0.57 while the odds of having poor glycemic control without DPN is.55. The relative risk being 1.03. DISCUSSION/SIGNIFICANCE OF IMPACT: Our population study revealed suboptimal glycemic control among a large set of patients in Western New York with a diagnosis of diabetes mellitus and a concurrent prescription for an opioid pain medication. A significant percentage of patients in our study population with a diagnosis of DPN might benefit in terms of decreased painful symptoms of neuropathy from monitoring and attempting to improve glycemic control. Additionally, in our patient population, there were no patients with diabetic peripheral neuropathy prescribed pregabalin or duloxetine, the first-line FDA-approved medications for painful DPN, Based on our population study, the quality of care for diabetic patients with DPN who are prescribed opioid pain medications should be monitored closely. First-line, FDA approved anticonvulsants and antidepressants should be considered for the treatment of painful symptoms when necessary. Attention should be directed towards monitoring and improving glycemic control in patients without DPN receiving opioid pain medications to attempt to prevent or delay the microvascular complications of diabetes, including the onset of painful peripheral neuropathy.

3048

\section{Measuring the Autonomic Nervous System for Translational Research: Identification of Non-invasive Methods}

Danielle Groat ${ }^{1}$, Ram Gouripeddi ${ }^{1}$, Yu Keui Lin ${ }^{1}$ and Julio C. Facelli ${ }^{1}$ ${ }^{1}$ The University of Utah School of Medicine

OBJECTIVES/SPECIFIC AIMS: The objective of this study is to identify and categorize non-invasive measurement methods for autonomic nervous system (ANS) symptoms that develop in hypoglycemic episodes. METHODS/STUDY POPULATION: We first reviewed literature for hypoglycemia symptomology. We then performed a selective literature review of Google Scholar, PubMed and Scopus for an ANS symptom and/or synonyms and the words 'sensor' or 'detection', e.g. 'sweat sensor' and 'tremor detection', studies utilizing non-invasive measurements in DM, and datasets of noninvasive measurements in DM. Measurement methods were then organized based on the ANS symptoms and existing metadata models for harmonizing sensors and surveys. RESULTS/ANTICIPATED RESULTS: We identified several measurement methods to for ANS symptoms during hypoglycemic events: thermometer, accelerometer, electrocardiogram (ECG), galvanic skin response (GSR), image processing, infrared imaging, thermal actuator, and ecological momentary assessment (EMA). The stage of implementation varied across the measurement methods from under development, to use in research and clinical settings, and even commercially available consumer products. Measurement methods that could be worn as wrist-band wearables or as film-based epidermal sensors would be capable of automatically gathering data with little to no effort required of the person wearing the device. Image-based methods would require the individual to actively engage in generating a photograph for analysis. In the case of EMA's, a message containing a question is sent to the individual, often via text message, soliciting short and immediate responses. It is anticipated that one sensor alone would not be sufficient to measure ANS responses to hypoglycemia, but rather several data points would be required. For example, if the GSR was the only signal, sweat in response to vigorous exercise or a warm environment would inject noise into the signal. Including the accelerometer data would allow for the identification of body movement which would indicate exercise, while an ECG signal could confirm the exercise. DISCUSSION/SIGNIFICANCE OF IMPACT: Impaired awareness of hypoglycemia (IAH) is a complication that develops in about $30 \%$ of type $1 \mathrm{DM}$ and $10 \%$ type $2 \mathrm{DM}$ populations. In individuals with intact awareness of hypoglycemia, the ANS leads to symptoms which includes: shaking, trembling, anxiety, nervousness, palpitation (i.e. change in heart rate and/or function), clamminess, sweating, dry mouth, hunger, pallor (i.e. drop in blood flow and/or skin-surface temperature), and pupil dilation. IAH is defined as the onset of hypoglycemia before the appearance of autonomic warning symptoms. IAH is caused by repeated exposures to low blood glucose levels, which reduces the body's ability to sense hypoglycemia, and therefore it is difficult for patients to recognize and self-treat. Individuals with IAH are six times more likely to experience severe hypoglycemia, an emergent condition which can lead to unconsciousness, seizure, coma, and death. Clinical investigators are developing interventions that aim to improve awareness of hypoglycemia. Surveys, observations by clinicians, and laboratory tests, often carried out in highly controlled in-patient settings, are currently used to assess the severity of IAH and the ANS's ability to respond to hypoglycemia. In other disease states, for example heart disease and Parkinson's disease, electrocardiograms and accelerometers have been used to assess heart function and tremor, respectively. However, there is currently a barrier to examining the efficacy of IAH interventions in real world settings as there are no established objective and non-invasive means to measure ANS symptoms due to hypoglycemia. This work encompasses the first important step necessary to direct translational researchers interested in testing the efficacy of IAH interventions and developing diagnostic tools for IAH in real-world studies outside the clinic. Next steps include evaluating these sensors and specifying EMA surveys, designing studies, and integration and assimilation of these data streams to identify true events of IAH by leveraging informatics platform such as the Utah PRISMS Informatics Ecosystem. Investigators would then be able to conduct studies that aim to develop and validate models that take sensor and EMA data as the input to detect and assess the severity of IAH.

3515

\section{Readability of online patient education materials on gynecologic malignancies from major medical associations}

David Samuel ${ }^{1}$, Nicole Vilardo ${ }^{1}$, Sara Isani ${ }^{1}$ and Gregory Gressel ${ }^{1}$

${ }^{1}$ Albert Einstein College of Medicine

OBJECTIVES/SPECIFIC AIMS: Patients are increasingly using online materials to learn about gynecologic cancer. Recent studies demonstrate that $85-96 \%$ of patients with a gynecologic malignancy 
utilize the Internet as a health resource. Providers can refer patients to educational materials produced by major medical associations available on their websites. However, patient educational materials (PEMs) published by professional organizations from other surgical specialties have been shown to be difficult to read for the average American. The NIH and AMA recommend that PEMs be written between a sixth and eighth grade reading level. In this study, we assess the readability of online PEMs on gynecologic cancer published by major medical associations. METHODS/STUDY POPULATION: Seven national medical association websites with PEMs on gynecologic malignancy were surveyed: American College of Obstetricians and Gynecologists, Center for Disease Control, Foundation for Women's Cancer, National Cancer Institute, National Cervical Cancer Coalition, National Ovarian Cancer Coalition, and Society of Gynecologic Oncology. Online PEMs were identified and analyzed using five validated readability indices. One-way ANOVA and Tukey's test were performed to detect differences in readability between publishers. RESULTS/ANTICIPATED RESULTS: Two hundred and thirty PEMs were included in this analysis. Mean readability grade levels with standard deviation were: 11.3 (2.8) for Coleman-Liau index; 11.8 (3.2) for Flesch-Kincaid; 11.1 (1.2) for FORCAST formula; 12.5 (2.7) for Gunning FOG formula; 12.1 (2.6) for New Dale-Chall formula; and 13.5 (2.5) for SMOG formula. Overall, PEMs were written at a mean $12^{\text {th }}$ grade reading level. Only $4.3 \%$ of articles were written at an $8^{\text {th }}$ grade reading level or below. ANOVA demonstrated a significant difference in readability between publishing associations $(\mathrm{p}<0.01)$. PEMs from the Center for Disease Control had a mean $10^{\text {th }}$ grade reading level and were significantly lower than all other organizations. PEMs from The Foundation for Women's Cancer had a mean $13^{\text {th }}$ grade reading level and were significantly higher than most other organizations. DISCUSSION/SIGNIFICANCE OF IMPACT: Gynecologic oncology PEMs available from major medical association are written well above the recommended sixth to eight grade reading level. Simplifying PEMs may improve patient understanding of their disease and facilitate physician-patient communication.

3081

Reducing Reintubation Risk in High-Risk Cardiac Surgery Patients with High Flow Nasal Cannula

Robert Edward Freundlich

Vanderbilt University Medical Center

OBJECTIVES/SPECIFIC AIMS: More than half a million adult patients nationally undergo cardiac surgery each year. Reintubation following cardiac surgery is common and associated with higher short- and long-term mortality, increased cost, and longer lengths of stay. The reintubation incidence is estimated at 5-10\%. Patients undergoing cardiac surgery are increasing in age and comorbidity burden, and receive increasingly complex cardiac surgical procedures, complicating decision making around when to extubate postoperative patients. Compounding this complexity are financial pressures to maintain high throughput and maximize ICU bed availability. Providers are often compelled to extubate high-risk patients earlier, despite the potential for an increased risk of reintubation. Understanding the risk factors for reintubation after cardiac surgery and identifying effective interventions to reduce these reintubations is of critical importance to optimize patient outcomes. High-flow nasal cannula (HFNC) provides up to 60 liters per minute of $100 \%$ oxygen, dead space washout, and humidification to improve secretion clearance, and has shown some benefits in improving hypoxia and reducing reintubation in select populations. However, its benefit in high-risk patients undergoing cardiac surgical procedures is not known and therefore clinicians may still be reluctant to extubate these patients early and introduce HFNC, despite the known risks of prolonged intubation. To address this important issue, we aim to develop and validate a model to predict postoperative reintubation after cardiac surgery using data readily available from the electronic health record (EHR) and use this data to complete a pilot randomized controlled trial (RCT) of postextubation HFNC to prevent reintubation in cardiac surgery patients identified as at high risk for reintubation. METHODS/STUDY POPULATION: Based on retrospective data demonstrating a $4.7 \%$ reintubation incidence within 48 hours in our CVICU, we estimate that there will be 340 reintubations available for analysis of the risk factors for reintubation to develop our predictive model from November 2, 2017 (our EHR go-live). We require 15 events per predictive variable to avoid overfitting the model, giving us at least 22 variables for analysis and inclusion in the model. Model validation and calibration will be performed using a bootstrapped validation cohort. Next, we will prospectively study 120 patients with a greater than $10 \%$ predicted risk of reintubation (double the baseline risk of the overall population) and randomly assign them to either HFNC or usual care, to test the hypothesis that HFNC decreases the rate of reintubation in high-risk patients. RESULTS/ANTICIPATED RESULTS: In addition to developing a predictive model, refining it, and validating its ability to predict the primary outcome of reintubation within 48 hours, I will further assess whether HFNC reduces total duration of mechanical ventilation, hospital length of stay, and ICU length of stay in this high-risk population. I will use these data to establish the feasibility of EHR-integrated predictive modeling and randomization, as well as to guide a future multicenter clinical trial that will pragmatically leverage the EHR for patient selection, enrollment, randomization, and data collection. DISCUSSION/ SIGNIFICANCE OF IMPACT: Assuming HFNC decreases reintubation rates by $50 \%$, at a 1:1 ratio of cases to controls, we will require 435 patients in each group ( 970 total), to have an $80 \%$ power and alpha of 0.05 to detect a difference. As this will require a multicenter study, we will instead focus on using data from this pilot study to: 1) refine our sample size estimates. 2) demonstrate the feasibility of our novel EHR-integrated pragmatic trial design. 3) identify and screen collaborators at other institutions, including obtaining important regulatory and legal approval. 4) establish a data safety monitoring board for the trial. 5) refine the data collection infrastructure, leveraging commercially available resources in one of the largest enterprise EHR systems (Epic) and associated resource-sharing products, such as Epic's App Orchard.

Systematically Integrating Microbiomes and Exposomes for Translational Research

Ram Gouripeddi ${ }^{1}$, Andrew Miller ${ }^{2}$, Karen Eilbeck ${ }^{2}$, Katherine Sward ${ }^{2}$ and Julio C. Facelli ${ }^{1}$

${ }^{1}$ The University of Utah School of Medicine and ${ }^{2}$ University of Utah; University of Utah; The University of Utah School of Medicine

OBJECTIVES/SPECIFIC AIMS: Characterize microbiome metadata describing specimens collected, genomic pipelines and microbiome results, and incorporate them into a data integration platform for enabling harmonization, integration and assimilation of microbial genomics with exposures as spatiotemporal events. METHODS/ STUDY POPULATION: We followed similar methods utilized in 